\title{
Glassy State and Cryopreservation of Mint Shoot Tips
}

\author{
Aline S. Teixeira \\ M. Elena González-Benito \\ Antonio D. Molina-García
}

Vitrification refers to the physical process by which a liquid supercools to very low temperatures and finally solidifies into a metastable glass, without undergoing crystallization at a practical cooling rate. Thus, vitrification is an effective freeze-avoidance mechanism and living tissue cryopreservation is, in most cases, relying on it. As a glass is exceedingly viscous and stops all chemical reactions that require molecular diffusion, its formation leads to metabolic inactivity and stability over time. To investigate glassy state in cryopreserved plant material, mint shoot tips were submitted to the different stages of a frequently used cryopreservation protocol (droplet-vitrification) and evaluated for water content reduction and sucrose content, as determined by ion chromatography, frozen water fraction and glass transitions occurrence by differential scanning calorimetry, and investigated by low-temperature scanning electron microscopy, as a way to ascertain if their cellular content was vitrified. Results show how tissues at intermediate treatment steps develop ice crystals during liquid nitrogen cooling, while specimens whose treatment was completed become vitrified, with no evidence of ice formation. The agreement between calorimetric and microscopic observations was perfect. Besides finding a higher sucrose concentration in tissues at the more advanced protocol steps, this level was also higher in plants precultured at $25 /-1^{\circ} \mathrm{C}$ than in plants cultivated at $25^{\circ} \mathrm{C}$.

\section{Introduction}

Biologically material, most frequently that being used for plant propagation (i.e., germplasm), can be stored at low temperature for long periods. Plant germplasm, particularly seeds, pollen, or reproductive tissues, is often kept at liquid nitrogen (LN) temperature to avoid deterioration. ${ }^{1}$ Cryopreservation is currently an important tool for germplasm longterm storage, requiring minimal space and maintenance, and the number of species and cultivars cryopreserved quickly increases. ${ }^{2}$ Low-temperature storage of living tissues and cells must avoid ice crystal formation (especially intracellular) during the whole process (cooling, storage, and final rewarming), as ice results lethal for cells, impairing specimens viability. ${ }^{3,4}$

Most techniques used include an initial step designed to reduce the tissues water content (with the limit of avoiding dehydration damage) and increase the cytoplasm internal viscosity. This step may include the incorporation of extrinsic viscosity enhancing substances (such as sugars, glycerol, or ethylene glycol) and the induction of cold adaptation and defense mechanisms by the plant cells. A second step consists of quick cooling by plunging into LN to avoid ice formation. The chances of ice crystallization are limited by both a decrease in the amount and mobility of intracellular water and an increase in cooling rates, hindering the rearrangement of water molecules into ice, a necessary previous nucleation step. Cooling to LN temperature places the system well into the glassy state. Once vitrified, despite some time-depending phenomena still taking place, relatively large molecular reorganizations, as those required for ice formation, are not possible and specimens can be stored for hypothetically indefinite periods, without ice being formed. ${ }^{5} \mathrm{~A}$ third step consists of the rewarming process, which must be performed quickly enough to avoid ice nucleation while crossing by the temperature region comprised between the glass transition temperature $\left(T_{\mathrm{G}}\right)$ and the equilibrium freezing point $\left(T_{\mathrm{f}}\right){ }^{6-8}$ Progressive dehydration and rapid cooling have been found to avoid the formation and growth of lethal intracellular ice in plant cells. ${ }^{9}$

Many apparently independent physical properties undergo large changes associated to the glass transition within similar temperature ranges. For example, the $T_{\mathrm{G}}$ values determined by calorimetry, thermal expansivity, or viscosity are often nearly identical. ${ }^{10}$ This is a result of all these properties 
being parallelly influenced by changes in molecular mobility occurring during the glass transition.

The initial works on mint cryopreservation by vitrification included gradual dehydration using a mixture of ethylene glycol, dimethyl sulfoxide (DMSO), and polyethylene glycol. ${ }^{1}$ A successful method among those usually used for plant germplasm cryopreservation is that named droplet-vitrification. ${ }^{11}$ It is derived from the droplet-freezing technique ${ }^{12}$ developed for preserving shoot tips, which are placed in droplets of cryoprotective medium and cooled slowly in a programmable freezer. In the droplet-vitrification method, the specimen is initially incubated in solutions with increasing concentration of cryoprotecting and dehydrating agents for different periods. Then it is treated and included in a small drop of cryoprotecting agents solution (containing high concentrations of glycerol, ethylene glycol, DMSO, and sucrose $^{13}$ ) and deposited on a small strip of aluminum foil. Finally, the strip with the droplets is quickly plunged into LN and then stored in a cryovial in a LN container. Warming is carried out similarly, plunging the strip plus droplets into warm liquid medium containing $1.2 \mathrm{M}$ sucrose and, after 20 min unloading, shoot tips are retrieved and placed on recovery medium. The results of this method, in terms of viability, have been good for a variety of systems. 2,11

The main advantage of this technique is enabling very high cooling and warming rates because of the small volume of medium in which the explants are placed. ${ }^{2}$ Many protocols involve exposure of plant shoot apices to PVS2 at $0^{\circ} \mathrm{C}^{13,14}$ To prevent cellular damage, cryoprotectant solutions both dehydrate and penetrate cells to stabilize proteins and membranes. However, the mode of action of each component may differ with cell type, species, temperature, and other solution components. ${ }^{15,16}$ A particular component of the cryoprotectant solutions used, DMSO, enhances chemical penetration into cells and, in spite of its own cytotoxicityfor which testing the treatment duration is essential-it has been found to promote survival of cryoexposed cells. ${ }^{8,17,18}$

The interplay between exogenous and endogenous carbohydrates plays an important role on plant resistance to stresses. For example, a two-step preculture treatment of gentian buds with increasing sucrose concentrations produced a raise of abscisic acid, followed by an increase of endogenous sucrose concentration, all of which resulting in a higher dehydration tolerance. ${ }^{19}$ Various high-performance liquid chromatography (HPLC) techniques have been developed to facilitate carbohydrate analyses in an effort to better understand their role in biochemical processes. High-performance ion chromatography (IC) coupled with pulsed amperometric detection (PAD) is an efficient method to quantify carbohydrates in natural samples and food products. ${ }^{20-24}$

Differential scanning calorimetry (DSC) is a well-known technique used for studying thermal properties of thermally driven processes. It has been used for long in cryopreservation studies, as it yields interesting information on freezing and vitrification processes. ${ }^{5,8,25-27}$ Water freezing latent heat is large, so calorimetry is a sensitive method to monitor ice formation or thawing, even in small quantities. Besides the amount of ice formed, after knowledge of the system's water content, the ratio of frozen-unfrozen water may also be obtained. DSC also yields $T_{\mathrm{f}}$, and its reduction speaks of increasing solute concentrations. $T_{\mathrm{G}}$ is also acquired, if scans are performed at sufficiently low temperature, although DSC is not particularly sensitive for this purpose.
Scanning electron microscopy (SEM) is a powerful and user-friendly microscopic technique, able to provide a wealth of structural information on a wide range of samples. When combined with a cryo-stage (cryo-SEM), it is especially interesting to study the microstructure of biological systems, not altered in sample preparation. ${ }^{28}$ Low-pressure LN frozen samples preserve most structural relations present in the original sample, with little or no artifacts. Cryo-SEM allows sample observation without need for prior chemical fixing or drying. Ice crystals formed are small and not altering tissue structures. Ice formed inside cells can be observed, after fracture in the cryo-stage and a suitable etching process, as black "void" regions surrounded by gray ridges formed by highly cryo-concentrated solution either frozen as euthectic, unfrozen, or even vitrified.

In this work, these three powerful techniques, DSC, cryoSEM, and HPLC, have been applied to follow the behavior toward LN cooling of specimens of plant germplasm-mint (Menta $\times$ piperitha) shoot tips-in different steps of the droplet-vitrification protocol. To date, no publication provides data obtained during this cryopreservation protocol, but only of its final outcome. Actually, very little physical information exists on actual cryopreserved or vitrified plant specimens. Besides, the combined use of information resulting from these physicochemical techniques allows us to better understand the phenomena taking place between the normal and the vitrified states of plant tissues and back to restore its viability as germplasm. Interesting relations among sucrose and cryoprotectant content in cells and its freezing and vitrification behavior are found, as well as with the other factors ruling over the physical control of ice formation: water content and cooling rate. The data presented here are expected to help to design improved cryopreservation procedures, involving less damaging cryoprotectants and/or suitable for problematic species.

\section{Materials and Methods}

\section{Plant material preculture and shoot tips extraction}

Shoot tips were extracted from in vitro shoots of Mentha $x$ piperita. In vitro plants were monthly subcultured on medium $\mathrm{MS}^{29}$ with $3 \%$ sucrose and incubated at constant temperature $\left(25^{\circ} \mathrm{C}\right)$ with a photoperiod of $16 \mathrm{~h}$ and an irradiance of $50 \mu \mathrm{mol} \mathrm{m} \mathrm{m}^{-2} \mathrm{~s}^{-1}$ from fluorescent tubes. One-node segments were obtained from these shoots, transferred to fresh medium, and incubated at alternating temperatures of $25^{\circ} \mathrm{C}$ (day) and $-1{ }^{\circ} \mathrm{C}$ (night), always with $16 \mathrm{~h}$ photoperiod and thermoperiod, $50 \mu \mathrm{mol} \mathrm{m}{ }^{-2} \mathrm{~s}^{-1}$ irradiance, provided by fluorescent tubes (stage a, see Figure 1). After 3 weeks of culture under these conditions, shoot tips (1-2 $\mathrm{mm})$ were excised from axillary buds.

\section{Incubation and dehydration of shoot tips}

A protocol based on that of Senula et al. ${ }^{11}$ was followed. Five excised shoot tips were precultured overnight with 2 $\mathrm{mL}$ of liquid MS medium containing $0.3 \mathrm{M}$ sucrose at $25^{\circ} \mathrm{C}$ over filter paper (stage b, see Figure 1). Thereafter, the explants were transferred to a Petri dish with $2 \mathrm{~mL}$ of loading solution ( $2 \mathrm{M}$ glycerol $+0.4 \mathrm{M}$ sucrose) over filter paper, for $20 \mathrm{~min}$, at room temperature (stage c). Finally, they were osmotically dehydrated in $2 \mathrm{~mL}$ PVS2 (plant vitrification solution 2: $30 \% \mathrm{w} / \mathrm{v}$ glycerol, $15 \% \mathrm{w} / \mathrm{v}$ ethylene glycol, $15 \%$ $\mathrm{w} / \mathrm{v}$ DMSO, and $0.4 \mathrm{M}$ sucrose in plant growth medium ${ }^{13}$ ) 
a: cold treatment

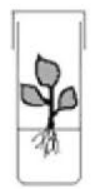

$$
\begin{aligned}
& \text { in vitro plants at } \\
& 25^{\circ} \mathrm{C} \text { (light) and }
\end{aligned}
$$$$
-1^{\circ} \mathrm{C} \text { (dark) }
$$

b: preculture

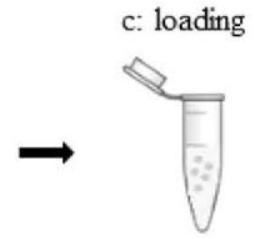

Overnight, in MS medium with $0.3 \mathrm{M}$ sucrose

\section{$20 \mathrm{~min}$ incubation in \\ $2 \mathrm{M}$ glycerol, $0.4 \mathrm{M}$ \\ sucrose}

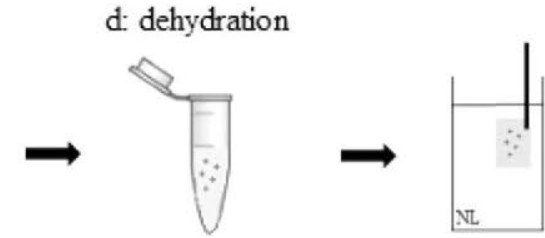

$20 \mathrm{~min}$ incubation

in PVS2 solution

Figure 1. Scheme of the steps of the droplet cryopreservation protocol used.

See text for more details on media and solutions used.

in a Petri dish, on filter paper, for $30 \mathrm{~min}$ at $0^{\circ} \mathrm{C}$ (stage d). Immersion in LN was carried out in the cryo-SEM or inside the aluminum pans of DSC, depending on the experiment. A schematic of these steps can be seen in Figure 1.

\section{Plant recovery and viability}

Treated and control shoot tips were warmed from LN temperature by plunging into warm liquid medium with $1.2 \mathrm{M}$ sucrose for $20 \mathrm{~min}$. After the unloading of cryoprotectants, shoot tips were cultured on regrowth medium, composed of solid MS medium supplemented with $0.5 \mathrm{mg} \mathrm{L}^{-1} \mathrm{BAP}+0.3$ $\mathrm{M}$ sucrose, cultivated in the dark for $24 \mathrm{~h}$ and finally placed into the culture room at $22-25^{\circ} \mathrm{C}$ and $16 \mathrm{~h}$ illumination. For control samples ( $-\mathrm{LN})$, all steps were carried out, including the incubation in PVS2 for $30 \mathrm{~min}$. After that the explants were rinsed in 1.2 M sucrose solution for $20 \mathrm{~min}$ and then placed on the regrowth medium.

Survival and regrowth were calculated as rates over the total number of shoot tips used. Survival was defined including all forms of visible viability (evidence of green structures or callus) observed 1-2 weeks after rewarming. Regrowth was defined as the formation of small plantlets, 4 weeks after rewarming.

\section{Low-temperature SEM}

Low temperature SEM (cryo-SEM) observations were performed with a Zeiss DSN 960 scanning microscope equipped with a Cryotrans CT-1500 cold plate (Oxford, UK). Three shoot tips in the same stage of the cryopreservation protocol were fitted on a special bracket with their axes vertically aligned. The geometry of sample and sample holder required optimization for both freezing and fracturing procedures: these steps require stable mechanical mounting of the sample on a support. This piece was plunged into LN under low pressure, physically fixing tissues for microscopic observation. This cooling step was considered equivalent to the cooling process taking place in the actual cryopreservation process by LN immersion. The holder was then inserted in the prechamber of the Cryotrans cold plate, at $-180^{\circ} \mathrm{C}$, and specimens were fractured perpendicularly to their axis to obtain a suitable observation surface (a cartoon of the tips before introduction in the microscope and after fracture can be seen in Figure 2). Later, samples were inserted in the microscope and etching (partial ice sublimation induced to provide contrast) was performed for 3 minutes at $-90^{\circ} \mathrm{C}$. Once etched and recooled, the sample was retrieved into the cryopreparation chamber and coated with high-purity $\mathrm{Au}$, which acts as a conductive contact for electrical charge. The
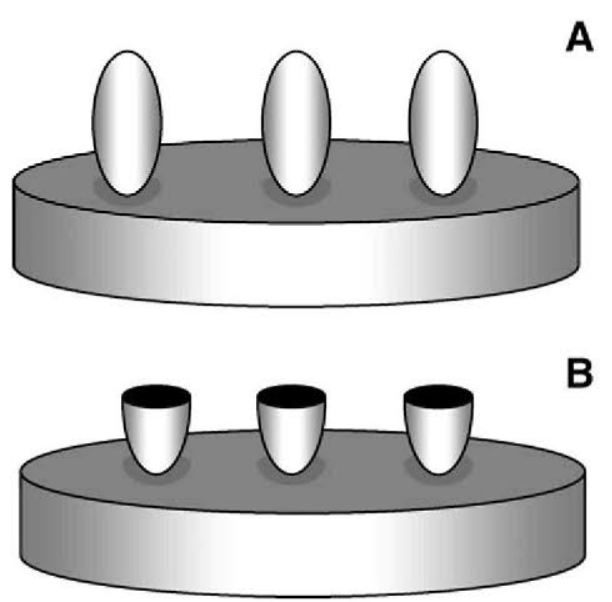

Figure 2. Cartoon showing the three mint tips on the microscopy holder before insertion in the microscope (A) and after equatorial freeze fracture $(B)$.

etched and coated sample was reinstated into the cooled SEM stage and observed using an accelerating voltage of 15 $\mathrm{kV}$ at $-150 /-160^{\circ} \mathrm{C}$.

\section{Dry matter and water content determination}

Six sets of five tips were used for determining the dry mater content $(\mathrm{dm})$ in shoot tips at the different stages of the cryopreservation protocol. It resulted from weighing after oven drying $\left(\sim 85^{\circ} \mathrm{C}, 72 \mathrm{~h}\right)$ and was expressed as relative to the total tip mass. The water content $\left(W_{c}\right)$ of shoot tips could be calculated for stages a and $b$, directly as the difference between its total mass and $\mathrm{dm}$. It was expressed as relative to the total shoot tip mass $\left(W_{\mathrm{c}(\mathrm{s})}\right)$ or the dry mass $\left(W_{\mathrm{c}(\mathrm{dm})}\right)$.

\section{Differential scanning calorimetry}

DSC experiments were performed with a Mettler-Toledo DSC 30 instrument (Mettler-Toledo, Griefsen, Switzerland). Cooling was performed using the calorimeter control $\left(10^{\circ} \mathrm{C}\right.$ $\min ^{-1}$ ), or for higher rates, by quenching the samples in LN (see below). The response of shoot tips apices to each step in the mint protocol was evaluated. Five shoot tips in the same stage of the cryopreservation protocol were introduced in a DSC pan, which was sealed and weighed. Shoot tips had been sequentially treated with MS medium, loading solution, and PVS2. Samples were submitted to a cooling scan from room temperature to $-150^{\circ} \mathrm{C}$ and a short $5 \mathrm{~min}$ equilibration at this temperature, followed by a warming scan (at 
$10^{\circ} \mathrm{C} \min ^{-1}$ ), back to room temperature. Calorimetric data were collected from two replicates per treatment.

For the quenching method shoot tips, sealed into a DSC pan, were quickly plunged into LN. Pans were subsequently cold-loaded into a prechilled DSC $\left(-150^{\circ} \mathrm{C}\right)$ for analysis.

Later, pans were punctured and dried in an oven at $85^{\circ} \mathrm{C}$, for $72 \mathrm{~h}$, when they showed constant weight. Thermograms were analyzed using the standard procedures provided in the Mettler-Toledo STARe software. Ice thawing thermal events (more precise than freezing events) were used for extraction of $T_{\mathrm{f}}$. The routine produced the temperature corresponding to the event onset, $T_{\mathrm{f} \text { (onset), corresponding to the equilibrium }}$ freezing temperature and that to the peak, $T_{\mathrm{f}(\text { peak) }}$.

The equilibrium freezing temperature can be related to the water activity $\left(a_{\mathrm{w}}\right)$ of the system to its osmolality $(\pi)$ and to its solute content. ${ }^{30,31}$ For comparative purposes, a "sucrose equivalent" concentration ( $\mathrm{Suc}_{\mathrm{eq}}$ ) and a "sodium chloride equivalent" $\left(\mathrm{NaCl}_{\mathrm{eq}}\right)$ were determined from $T_{\mathrm{f}}$, assuming that the only solute present was sucrose or sodium chloride, respectively. $T_{\mathbf{f} \text { (peak) }}$ data were used for the determination of these parameters, as it was considered to better represent the behavior of the whole phase change. Equation $(1)^{30}$ was followed for $a_{\mathrm{w}}$ determination:

$$
\ln a_{\mathrm{w}}=\Delta H_{\mathrm{f}(\mathrm{w})} A_{\mathrm{w}}\left(T_{\mathrm{f}}-T_{\mathrm{f}} *\right) /\left(T_{\mathrm{f}} T_{\mathrm{f}} *\right),
$$

where $\Delta H_{\mathrm{f}(\mathrm{w})}$ is the pure water freezing enthalpy (specific, per water gram), $A_{\mathrm{w}}$ is the water molecular weight, and $T_{\mathrm{f}}{ }^{*}$ is the equilibrium freezing point of pure water. The osmolality was calculated using Eq. $2^{31}$ :

$$
\pi=\Delta T_{\mathrm{f}} / 1.86,
$$

where $\Delta T_{\mathrm{f}}$ is the freezing point depression, which for water at atmospheric pressure, being the pure substance freezing point $0^{\circ} \mathrm{C}$, equals to $T_{\mathrm{f}}$ with a positive sign.

$\mathrm{Suc}_{\mathrm{eq}}$ and $\mathrm{NaCl}_{\mathrm{eq}}$ could not be calculated from Raoult's law, using analytical equations, ${ }^{32}$ and only valid for more diluted solutions. Experimental data from literature ${ }^{30,33}$ and calorimetric determinations performed on concentrated sucrose solutions (data not shown) were used to relate these hypothetic solute concentrations with the observed cryoscopic temperature decrease.

The process-associated enthalpy, phase change enthalpy $\Delta H_{\mathrm{f}}$, proportional to the event area, was also obtained. It was expressed as relative to either the total shoot tip mass $\left(\Delta H_{\mathrm{f}(\mathrm{s})}\right)$, its dry mass $\left(\Delta H_{\mathrm{f}(\mathrm{dm})}\right)$, or its water content $\left(\Delta H_{\mathrm{f}(\mathrm{w})}\right)$ for allowing easier data comparison.

Water fractions: frozen water $\left(W_{\mathrm{f}}\right)$ and unfrozen water $\left(W_{\mathrm{u}}\right)$ were calculated by comparison of $\Delta H_{\mathrm{f}}$ and the pure water freezing enthalpy $\left(\Delta H_{\mathrm{f}(\mathrm{w})}=333.4 \mathrm{~J} \mathrm{~g}^{-1}\right.$ at $\left.0^{\circ} \mathrm{C}\right)$, together with the total water contents previously determined, using Eq. (3):

$$
W_{\mathrm{f}}=\left(\Delta H_{\mathrm{f}} / \Delta H_{\mathrm{f}(\mathrm{w})}\right) ; W_{\mathrm{u}}=W_{\mathrm{c}}-W_{\mathrm{f}} .
$$

Specific enthalpy values (per sample gram) were always used after the corresponding oven dry pan weighs. Enthalpy values measured at temperatures different from the corresponding phase change equilibrium temperature, $0^{\circ} \mathrm{C}$, are underevaluated, as a result of the difference between water and ice heat capacities $\left(C p_{w}\right.$ and $C p_{h}$, respectively). So, the value of $\Delta H_{\mathbf{f}(\mathrm{w})}$ was corrected using Eq. (4), before applying Eq. (3). Single freezing temperatures, $T_{\mathrm{f}(\text { peak) }}$, were used, and $C p_{w}$ and $C p_{i}$ data were obtained from a data calculation routine produced in this laboratory, ${ }^{34}$ after interpolation when required, and other sources. ${ }^{35,36}$

$$
\Delta H f \operatorname{corr}=\Delta H \mathrm{f}-\left(\int_{0}^{T_{\mathrm{f}}} \mathrm{Cp}_{\mathrm{w}} \mathrm{dT}-\int_{T_{\mathrm{f}}}^{0} \mathrm{Cp}_{\mathrm{j}} \mathrm{dT}\right)
$$

Frozen $\left(W_{\mathrm{f}}\right)$ and unfrozen $\left(W_{\mathrm{u}}\right)$ water contents were expressed as relative to the total shoot tip mass $\left(W_{\mathrm{f}(\mathrm{s})}\right.$ and $\left.W_{\mathrm{u}(\mathrm{s})}\right)$, its dry mass $\left(W_{\mathrm{f}(\mathrm{dm})}\right.$ and $\left.W_{\mathbf{u}(\mathrm{dm})}\right)$, or its total water content, when available, $\left(W_{\mathrm{f}(\mathrm{w})}\right.$ and $\left.W_{\mathrm{u}(\mathrm{w})}\right)$.

The glass transition can be observed as a small step in the heat capacity thermogram baseline. The corresponding heat capacity increment is very small and, in our experiments, only could be appreciated by using 30 shoot tips in the same DSC pan.

For comparative purposes, calorimetric experiments were performed with samples of the cryoprotectant solutions used in each stage of the droplet protocols (i.e., liquid MS medium with $0.3 \mathrm{M}$ sucrose, loading solution, and PVS2). Experiments were performed in the same conditions than those carried out with shoot tip specimens.

\section{Sucrose content assessment in shoot tips}

Specimens were analyzed after each stage of the cryopreservation procedure for its sucrose content. Five shoot tips were weighed (average fresh weight $3.0 \pm 0.1 \times 10^{-3} \mathrm{~g}$ ) and mortar-ground into a fine powder in LN. As grinding proceeded, the vessel was slowly warmed. Immediately, 1.5 $\mathrm{mL}$ Milli-Q water was added and the mixture was filtered through a syringe-driven filter (Millex-GS, $0.22 \mu \mathrm{m}$ ).

The determination of sucrose was carried out by an Ion Chromatography 817 Bioscan (Metrohm, Herisau, Switzerland) system equipped with a Metrosep Carb 1-250 column and a PAD with a gold electrode. A three-step PAD protocol was used with the following time intervals ( $\mathrm{ms}$ ) and potentials $(\mathrm{mV}): \quad t 1, \quad 400 / E 1=+0.05 \quad$ (detection); $t 2, \quad 200 /$ $E 2=+0.75$ (cleaning); and $t 3,400 / E 3=-0.15$ (regeneration). Samples $(1.5 \mathrm{~mL})$ were injected using an autosampler (model, 838 Advanced Sample Processor, Metrohm, Herisau, Switzerland) and the flow rate through the column was $1 \mathrm{~mL}$ $\min ^{-1}$ and the eluent was $100 \mathrm{mM} \mathrm{NaOH}$.

Identification of sucrose was performed by comparison with the retention time of the pure standard sucrose (Merck) and the data were acquired using IC Net 2.3 software. Sucrose content was expressed as ppm and the data were the mean of three replicates. Results (Suc) are expressed as ppm of the total tip mass.

\section{Results and Discussion}

\section{Detection of glassy state and ice by cryo-SEM}

Two different observation approaches were compared: secondary electron and backscattered electron image. Basically, the same information was obtained with both; however, the cellular structures could be better appreciated using backscattered electrons, and this was used in the observations and micrographs presented here.

Figure 3 shows the micrographs showing shoot tips cooled in LN in the microscope cryounit as described in the Materials and Methods section, in different moments of the droplet cryopreservation protocol: control (A); stage a, cold treatment $(\mathrm{B})$; stage $\mathrm{b}$, preculture $(\mathrm{C}, \mathrm{D})$; stage $\mathrm{c}$, loading $(\mathrm{E}, \mathrm{F})$; and stage $\mathrm{d}$, dehydration $(\mathrm{G}, \mathrm{H})$. Micrographs $\mathrm{C}, \mathrm{E}$, and $\mathrm{G}$ 

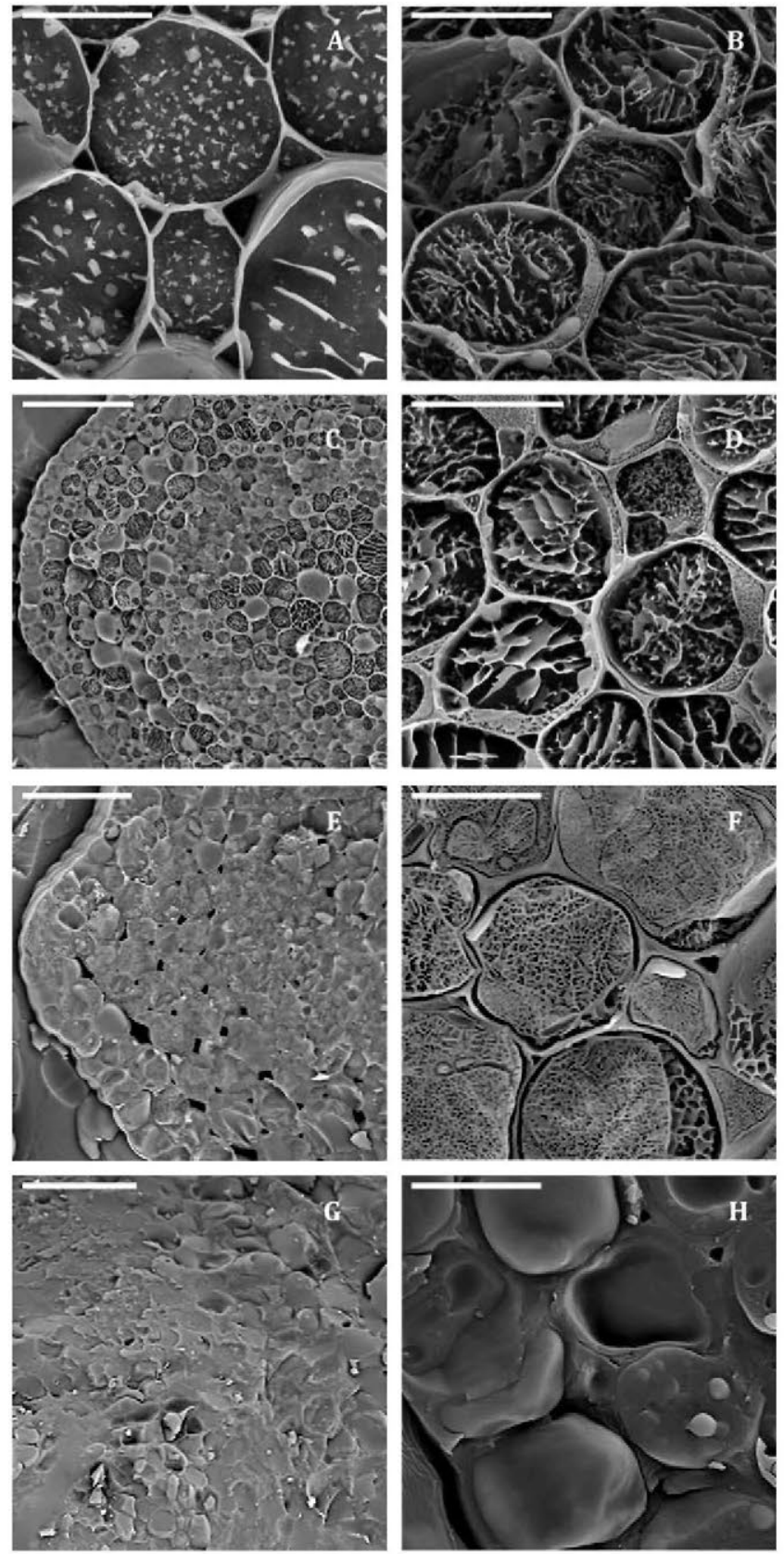

Figure 3. Cryo-SEM micrographs of shoot tips cooled in $\mathrm{LN}$ in the microscope cryounit (see Materials and Methods).

Control (A); stage a, cold treatment (B); stage b, preculture (C and $\mathrm{D})$; stage c, loading ( $\mathrm{E}$ and $\mathrm{F})$; and stage $\mathrm{d}$, dehydration $(\mathrm{G}$ and $\mathrm{H})$. The bar corresponds to $10 \mu \mathrm{m}(\mathrm{A}, \mathrm{B}, \mathrm{D}, \mathrm{F}$, and $\mathrm{H})$ or 50 $\mu \mathrm{m}(\mathrm{C}, \mathrm{E}$, and $\mathrm{G})$.

show lower magnification images of the shoot tips axial plane. The concentric cell distribution in the equatorially fractured tips could be appreciated. There were no noticeable differences among central or more external cells, indicating that the fracture was close to the apical dome, so effective homogeneity for temperature and solute and water concentrations could be assumed for tip cells.

Ice crystals formed in the more concentrated solutions were smaller because of the limitations to crystal growth caused by the higher solution viscosity. On the other hand, conversion of water into ice during cooling increased the concentration of solutes in the more concentrated regions. The etching procedure applied gave rise to a contrast in the
SEM micrograph, removing part of the ice formed by sublimation and leaving the darker regions separated by sector or "ridges" of freeze-concentrated solution that can be appreciated in micrographs $\mathrm{A}-\mathrm{F}$. This behavior allowed visualization of individual crystals. Sublimation is only taking place at an appreciable rate out of pure ice crystals, whereas water in glassy state has a sublimation rate almost negligible. ${ }^{37,38}$ So, when solutions become vitrified, they cannot be etched in this way. Micrographs $\mathrm{G}$ and $\mathrm{H}$ show a complete lack of the structural details revealed by etching.

\section{Determination of preculture effect by cryo-SEM}

Figures $3 \mathrm{~A}, \mathrm{~B}$ show, respectively, typical cryo-SEM micrographs after 3 weeks of culture at $25^{\circ} \mathrm{C}$ (control apices) or at $25 /-1^{\circ} \mathrm{C}$. Widespread cellular disruption occurred in control apices, as it can be observed in micrograph $3 \mathrm{~A}$, where ice can be seen as dark areas inside the cell's cytoplasm and vacuole; meanwhile, clearer parts would correspond to the supercooled cryoconcentrated solution matrix. Cold-treated shoot tips (3B) showed the formation of smaller ice crystals, mostly in the vacuole space, which indicates a higher solute cytoplasmic concentration with respect to control apices. The observed size crystal reduction is understood because of an increase in the amount of endogenous cryoprotecting agents developed by the plant during the cold hardening procedure, confirming the positive influence of tips cold hardening before cryopreservation observed by other workers. ${ }^{11}$

\section{Observed plant recovery and viability}

Both survival and recovery reached a $96 \%$ value for specimens treated with the complete droplet cryopreservation protocol (i.e., quenched in LN after stage d, dehydration in PVS2). Meanwhile, survival and recovery of previous stages, a to $\mathrm{c}$, were $0 \%$ for all cases. The control samples (-LN) showed a $100 \%$ survival and recovery.

\section{Analysis of the droplet-vitrification protocol steps by cryo-SEM}

Micrographs of specimens in the stages b (preculture) (C, D), c (loading) (E, F), and d (dehydration) $(\mathrm{G}, \mathrm{H})$ of the droplet-vitrification protocol can be seen in Figure 3. Specimens in both $\mathrm{b}$ and $\mathrm{c}$ stages showed a clearly visible tissular and cellular structure, with some organelles and vacuolar membrane elements present. Specimens in stage b showed larger ice crystals and a higher degree of alteration of cellular structures by ice than those in stage c. Tips treated with alternating temperatures (see previous section) had a similar aspect to those in step b (preculture). The ice crystal size difference could be due to concentration differences among samples, as the crystals formed in the more diluted solution can grow to a larger final size, whereas those occurring in concentrated solutions, where molecular mobility is impaired, would be smaller.

During the treatment in the loading solution, the cells of specimens in stage $\mathrm{c}$ of the protocol accumulated exogenous glycerol and other solutes of endogenous origin. These cells were clearly partly plasmolyzed as a result of the osmotic dehydration imposed by the loading treatment, which was related to the progressive acquisition of tolerance toward freezing during the protocol.

PVS2, the cryopreservation solution used in the last step, affected cellular freezing properties. ${ }^{8}$ It has components that 
Table 1. Compositional and Thermal Parameters of the Thawing Events Obtained from DSC Thermograms for Mint Shoot Tips Specimens at Different Stages (a-d) of the Droplet Cryopreservation Protocol

\begin{tabular}{|c|c|c|c|c|}
\hline & $\begin{array}{l}\text { Cold Treatment } \\
\text { (Stage a) }\end{array}$ & $\begin{array}{l}\text { Preculture } \\
\text { (Stage b) }\end{array}$ & $\begin{array}{l}\text { Loading } \\
\text { (Stage c) }\end{array}$ & $\begin{array}{l}\text { Dehydration } \\
\text { (Stage d) }\end{array}$ \\
\hline $\mathrm{dm}\left(\mathrm{g}_{\mathrm{dm}} \mathrm{g}_{\mathrm{sample}}{ }^{-1}\right)$ & $0.117 \pm 0.009$ & $0.151 \pm 0.009$ & $0.344 \pm 0.049$ & $0.252 \pm 0.013$ \\
\hline$W_{\mathrm{c}(\mathrm{s})}\left(\mathrm{g}_{\text {water }} \mathrm{g}_{\text {sample }}{ }^{-1}\right)$ & $0.883 \pm 0.009$ & $0.849 \pm 0.009$ & - & - \\
\hline$W_{\mathrm{c}(\mathrm{dm})}\left(\mathrm{g}_{\text {water }} \mathrm{g}_{\mathrm{dm}}-1\right)$ & $7.56 \pm 0.65$ & $5.64 \pm 0.39$ & - & - \\
\hline$T_{\mathrm{f} \text { (onset) }}\left({ }^{\circ} \mathrm{C}\right)$ & $-2.96 \pm 0.06$ & $-4.9 \pm 1.8$ & $-7.93 \pm 0.79$ & - \\
\hline$T_{\text {f(peak) }}\left({ }^{\circ} \mathrm{C}\right)$ & $-0.48 \pm 0.08$ & $-0.58 \pm 0.21$ & $-5.5 \pm 1.1$ & - \\
\hline$\Delta H_{\mathrm{f}(\mathrm{s})}\left(\mathrm{J}_{\mathrm{sample}}{ }^{-1}\right)$ & $241 \pm 16$ & $209.85 \pm 0.12$ & $93.0 \pm 7.2$ & - \\
\hline$\Delta H_{\mathrm{f}(\mathrm{dm})}\left(\mathrm{J} \mathrm{g}_{\mathrm{dm}}{ }^{-1}\right)$ & $1,800 \pm 650$ & $1,394 \pm 83$ & $274+60$ & - \\
\hline$\Delta H_{\mathrm{f}(\mathrm{w})}\left(\mathrm{J} \mathrm{g}_{\mathrm{w}}{ }^{-1}\right)$ & $272 \pm 14$ & $247.1 \pm 2.5$ & - & - \\
\hline$W_{\mathrm{f}(\mathrm{s})}\left(\mathrm{g}_{\text {water }} \mathrm{g}_{\text {sample }}{ }^{-1}\right)$ & $0.724 \pm 0.47$ & $0.632 \pm 0.000$ & $0.290 \pm 0.023$ & 0 \\
\hline$W_{\mathrm{f}(\mathrm{dm})}\left(\mathrm{g}_{\text {water }} g_{\mathrm{dm}}-1\right)$ & $6.20 \pm 0.88$ & $4.20 \pm 0.25$ & $0.86 \pm 0.19$ & 0 \\
\hline$W_{\mathrm{f}(\mathrm{w})}\left(\mathrm{g}_{\text {water }} \mathrm{g}_{\text {water }}{ }^{-1}\right)$ & $0.820 \pm 0.045$ & $0.744 \pm 0.007$ & - & 0 \\
\hline$W_{\mathrm{u}(\mathrm{s})}\left(\mathrm{g}_{\text {water }} \mathrm{g}_{\text {sample }}{ }^{-1}\right)$ & $0.159 \pm 0.038$ & $0.217 \pm 0.009$ & - & - \\
\hline$W_{\mathbf{u}(\mathrm{dm})}\left(\mathrm{g}_{\text {water }} \mathrm{g}_{\mathrm{dm}}-1\right)$ & $1.34 \pm 0.22$ & $1.44 \pm 0.14$ & - & - \\
\hline$W_{\mathrm{u}(\mathrm{w})}\left(\mathrm{g}_{\text {water }} \mathrm{g}_{\text {water }}-1\right)$ & $0.180 \pm 0.045$ & $0.256 \pm 0.007$ & - & - \\
\hline$a_{\mathrm{w}}$ & $0.9954 \pm 0.001$ & $0.9944 \pm 0.002$ & $0.9470 \pm 0.001$ & - \\
\hline$\pi(\mathrm{Osm})$ & $0.26 \pm 0.04$ & $0.31 \pm 0.12$ & $2.97 \pm 0.60$ & - \\
\hline
\end{tabular}

are penetrating (dimethyl sulfoxide, glycerol, and ethylene glycol) and nonpenetrating (sucrose) for the cellular membrane of most cell types. ${ }^{39}$ This complex solution served to dehydrate shoot tips and to change the behavior of the water remaining within shoot tips.

Micrographs of specimens in the stage $d$ of the protocol, after osmotic dehydration in PVS2 solution, presumably with a lower water content and more concentrated in solutes induced by permeation from the cryopreservation solution, showed a gray continuous, little detailed surface, indicating vitrification (Figures 3G,H).

\section{Changes in the dry mass and water content during the droplet-vitrification protocol}

The dry mass and water content of shoot tips, derived from its weight difference after drying, are shown in Table 1. For step a and b, where water was the only volatile substance (overseeing very small quantities of organic compounds), $W_{\mathrm{c}}$ could be obtained by this procedure and is presented in Table 1, expressed as related to the total sample mass $W_{\mathrm{c}(\mathrm{s})}$ or to the dry mass $W_{\mathrm{c}(\mathrm{dm})}$. The water content of tips undertook a small decrease with the first steps of the cryopreservation protocol, associated to sucrose incubation in stage $b$. Water content for stages $c$ and $d$ could not be derived from oven-drying data, as the amount of volatile substances apart from water was high. Nevertheless, it could be assumed that there was a large decrease in the water content associated to step c, as inferred from the increase in $\mathrm{dm}$, which was in good agreement with the reported microscopic observations showing a smaller ice crystal size in these samples and with the sucrose content data (see below).

Stage d, following the same arguments, would show the complex result of water osmotically driven out of the cell by the strong potential of the very concentrated PVS2 solution, rich in the nonpenetrating sucrose, entrance of the penetrating agents DMSO, ethylene glycol, and glycerol, and water retention inside the cell by the increased internal presence of these cryoprotectors, ${ }^{8,39-42}$ notwithstanding the increasing presence of endogenous cryoprotecting (and often water binding) agents, as a result of the plant response to the induced stress.

\section{Measurement of ice heat of fusion using DSC}

Figure 4 shows typical rewarming DSC thermograms for the same specimens types (at stages a-d) previously cooled

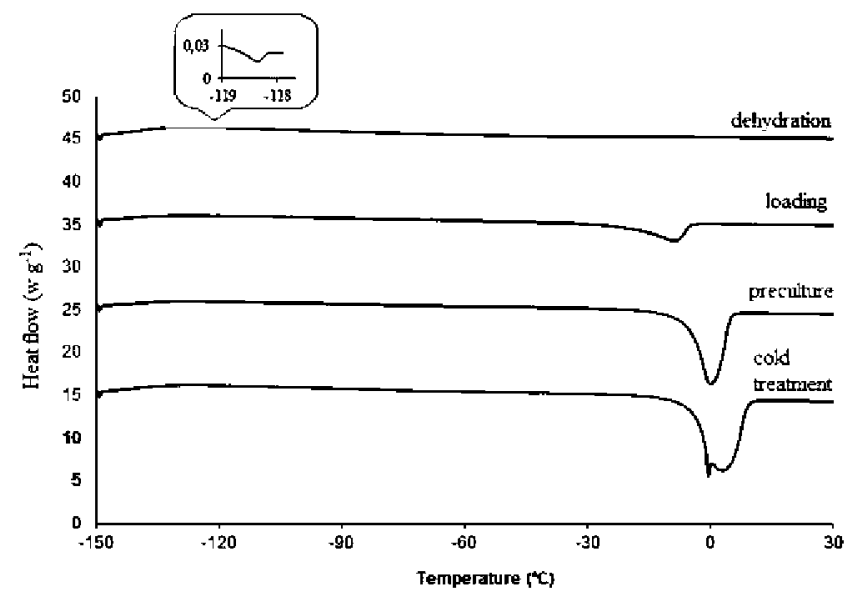

Figure 4. Typical DSC thermograms corresponding to rewarming processes of shoot tips in different stages of the droplet cryopreservation protocol: cold treatment (stage a), preculture (stage b), loading (stage c), and dehydration (stage d).

Each experiment was performed with five shoot tips. The insert shows an expanded thermogram section which allows the glass transition, obtained with 30 tips, to be appreciated. Scanning rate was $10^{\circ} \mathrm{C} \mathrm{min}{ }^{-1}$ (See Materials and Methods and Figure 1 for more details). The ordinates scale of the thermograms baseline is arbitrary.

at a rate of $10^{\circ} \mathrm{C} \min ^{-1}$. Table 1 shows the thermal parameters derived from DSC experiments, $T_{\mathrm{f} \text { (onset) }}$ and $T_{\text {f(peak) }}$, respectively, the onset temperature of the melting endotherm (corresponding to the equilibrium freezing temperature) and its peak temperature. The freezing enthalpy $(\Delta H)$ is also included. The information that both enthalpy and equilibrium freezing temperature can provide is of the highest interest, as it yields information on the water content within cells and, in a context where it is difficult to know the detailed cytoplasm composition and interacting effects of endogenous and exogenous solutes, to draw conclusions on the availability of this water, to form ice, to permit diffusion-driven processes and even to keep the stability of protein and membranes. Specimens both in stages $b$ and $c$ of the cryopreservation protocol showed freezing (not shown) and melting events in the respective cooling and rewarming scans.

The equilibrium freezing temperature is a good raw data to determine accurately solution characteristics, such as water activity and frozen and unfrozen water. ${ }^{32}$ However, 
the precision yielded by DSC for melting temperatures, especially in complex systems, such as natural tissues, is limited. The onset temperature is considered usually as the best estimation of the equilibrium freezing point. But, in complex solutions, compartmentalized in different isolated pools, with markedly different composition and water contents (cytoplasm, vacuoles, intercellular space, different cells, and tissues) the onset temperature would rather correspond to the melting of the most concentrated pool, which would thaw at the lowest temperature.

We have considered that, for these sample types, the peak temperature was giving a better estimation of the average melting of the specimens studied. Moreover, the DSC-measured temperature was affected by the thermal differences (as well as the compositional ones) within the sample, not negligible at a relatively fast scanning speed, as $10^{\circ} \mathrm{C} \mathrm{min}^{-1}$.

The temperatures determined were decreasing steeply with the progress of the cryopreservation protocol, which spoke about the increase in the "number" of solute molecule concentration. The last stage showed no thawing event, and there would be no frozen water at all in these conditions. From $T_{\mathrm{f}}$, the aqueous solution parameters $a_{\mathrm{w}}$ and $\pi$ were derived and shown in Table 1.

The thawing enthalpy is, in practice, a much more accurate parameter than freezing enthalpy, depending less on kinetic, geometric, or compartmentalization issues. The phase change enthalpy, which is presented in Table 1 as relative to either the total sample mass, $\Delta H_{\mathrm{f}(\mathrm{s})}$, the dry mass, $\Delta H_{\mathrm{f}(\mathrm{dm})}$, or the total water content, $\Delta H_{\mathrm{f}(\mathrm{w})}$, decreased as the protocol progresses. After correction for the different values of the pure water melting enthalpy at the actual phase change temperature for each of these processes, the amount of thawed water in each process could be calculated by simple comparison with this quantity. The resulting frozen water content, presented in Table 1 relative to either the total sample mass, $W_{\mathrm{f}(\mathrm{s})}$, the dry mass, $W_{\mathrm{f}(\mathrm{dm})}$, or the total water content, $W_{\mathrm{f}(\mathrm{w})}$, again markedly decreased as the protocol progressed. The last step could also be comprised in this trend, as $W_{\mathrm{f}}$ would equal to zero.

The unfrozen water fraction (derived from the frozen water fraction) can help to visualize the changes undergoing in the cryopreserved tissues, and is also shown in Table 1 relative to the total sample mass, $W_{\mathrm{u}(\mathrm{s})}$, the dry mass, $W_{\mathrm{u}(\mathrm{dm})}$, or the total water content, $W_{\mathrm{u}(\mathrm{w})}$. Traditionally, frozen water was understood and often named as free water, whereas the unfrozen fraction was considered as bound water. ${ }^{43}$ These denominations are much criticized nowadays, ${ }^{44}$ as the differences found in the actual binding of these two water fractions are very small, if any at all. ${ }^{45-50}$

For comparative purposes, Figure 5 shows typical calorimetric thermograms obtained during rewarming for the cryoprotective solutions used (at stages b-d), in the same conditions than those applied in the experiments of Figure 4. $\Delta H_{\mathrm{f}(\mathrm{s})}$ for stage b (liquid MS medium with $0.3 \mathrm{M}$ sucrose) was $228 \pm 2 \mathrm{~J} \mathrm{~g}^{-1}$ sample ${ }^{-1}$ whereas for stage c (loading solution) it was $138 \pm 2 \mathrm{~J} \mathrm{~g}_{\text {sample }}{ }^{-1}$. Stage d (PVS2) showed no melting exotherm. $\Delta H_{\mathrm{f}(\mathrm{w})}$ for stage b amounted to $250 \pm 3 \mathrm{~J}$ $\mathrm{g}_{\mathrm{w}}{ }^{-1}$. The $T_{\mathrm{f}(\text { onset })}$ were $-3.97^{\circ} \mathrm{C} \pm 0.2^{\circ} \mathrm{C}$ for stage $\mathrm{b}$ and $-4.68^{\circ} \mathrm{C} \pm 0.3^{\circ} \mathrm{C}$ for stage $\mathrm{c}$.

\section{Effects of quench-cooling on heat of fusion of ice}

In Figure 6, thermograms of the rewarming DSC stage of mint shoot tips cooled by quenching are compared with

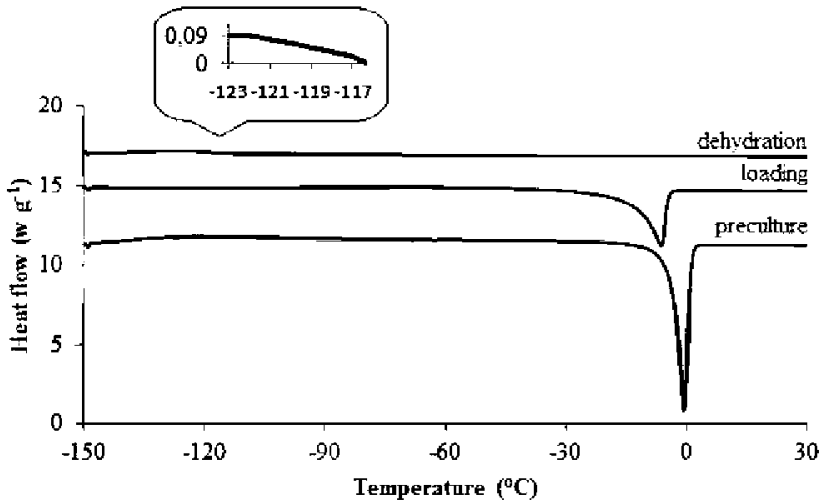

Figure 5. Typical DSC thermograms corresponding to rewarming processes of the cryopreservation solutions used in different stages of the droplet cryopreservation protocol: preculture (stage b): liquid MS medium containing $0.3 \mathrm{M}$ sucrose, loading (stage c): loading solution, and dehydration (stage d): PVS2.

The insert shows an expanded thermogram section which allows the glass transition to be appreciated. Scanning rate was $10^{\circ} \mathrm{C} \mathrm{min}{ }^{-1}$ (See Materials and Methods and Figure 1 for more details). The ordinates scale of the thermograms baseline is arbitrary.

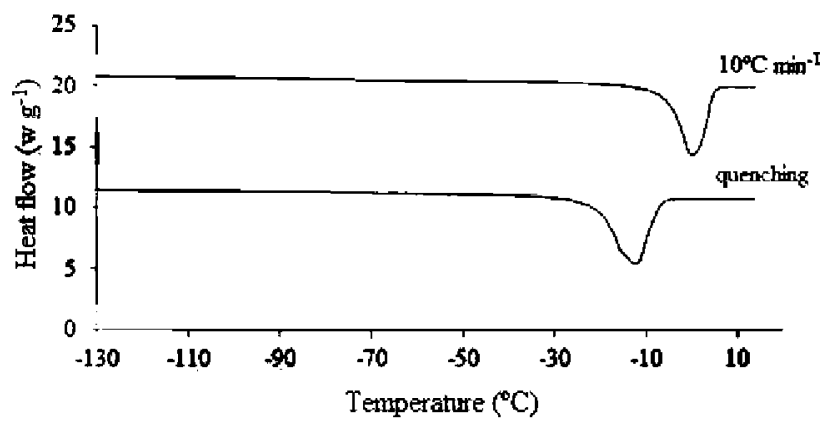

Figure 6. DSC scans showing warming at $10^{\circ} \mathrm{C} \mathrm{min}^{-1}$ for shoot tips treated at the preculture stage (b) of the droplet cryopreservation protocol.

Cooling rate was $10^{\circ} \mathrm{C} \mathrm{min}^{-1}$ or quenching (direct immersion of the DSC pan in LN).

those at a rate of $10^{\circ} \mathrm{C} \min ^{-1}$ in specimens of step a. It can be seen that, in the quench cooled sample, the thawing temperature decreased and the enthalpy increased slightly. These results, although consistent in all repeats and for experiments in different conditions (data not shown), were unexpected, and of difficult explanation. A possibility is that the fast cooling might trap solute molecules within the ice phase, which would later contribute to an average effective melting at lower temperatures. ${ }^{51-53}$

Nevertheless, this behavior, although may be further from equilibrium than those observed in the slow cooling, would correspond more closely to the real events taking place in cryopreservation practice.

\section{Detection of glass transition by DSC}

Specimens at the last stage of the cryopreservation protocol did not show any freezing event, in any case, either at the cooling (not shown) or melting runs of DSC studies. Nevertheless, in many experiments, the step in the heat capacity baseline showing the actual glass transition in DSC 
Table 2. Solute Content of Mint Shoot Tips at the Different Stages of the Droplet Cryopreservation Protocol

\begin{tabular}{lcccc}
\hline & $\begin{array}{c}\text { Cold Treatment } \\
\text { (stage a) }\end{array}$ & $\begin{array}{c}\text { Preculture } \\
\text { (stage b) }\end{array}$ & $\begin{array}{c}\text { Loading } \\
\text { (stage c) }\end{array}$ & $\begin{array}{c}\text { Dehydration } \\
\text { (stage d) }\end{array}$ \\
\hline $\operatorname{Suc}_{(\mathrm{s})}\left(\mathrm{ppm}_{\text {total sample weight }}\right)$ & $5,900 \pm 600$ & $6,500 \pm 300$ & $46,900 \pm 700$ & $53,000 \pm 4,000$ \\
$\operatorname{Suc}_{(\mathrm{dm})}\left(\mathrm{g} \mathrm{g}_{\mathrm{dm}}{ }^{-1}\right)$ & $0.059 \pm 0.006$ & $0.044 \pm 0.002$ & $0.137 \pm 0.002$ & $0.209 \pm 0.015$ \\
$\mathrm{Suc}_{\mathrm{eq}}(\mathrm{ppm})^{*}$ & 8,800 & 10,800 & 470,000 & - \\
$\mathrm{NaCl}_{\text {eq }}(\mathrm{ppm})^{*}$ & 8,000 & 10,000 & 91,000 & -
\end{tabular}

*These concentrations, as derived from the freezing point depression, refer to the solution mass (mass of solutes plus mass of solvent), not taking into consideration any nondissolved substances.

thermograms could not be appreciated. Cooling rates of $10^{\circ} \mathrm{C}$ min $^{-1}$, controlled by the calorimeter itself, or externally driven, by quick introduction into LN (quenching) were tested, but the glass transition could only be observed (for both cooling rates) for samples containing 30 shoot tips. Calorimetry is often claimed to be a rather insensitive method to observe the glass transition, ${ }^{54}$ and this is especially applicable here, as the dehydrated tips have a very small weight (total average sample mass for each DSC experiment-five tips-for d-stage specimens was $\sim 3.5 \mathrm{mg}$ and the total mass of water in these samples was $2.5 \mathrm{mg}$ ).

As shown in the insert of Figure 5, PVS2 solution is also showing a glass transition in these conditions, centered at $-120^{\circ} \mathrm{C}$.

\section{Changes in sucrose content during the droplet-vitrification protocol}

The actual composition of the cellular contents in cryopreservation conditions is critical for the formation of ice or the vitrification of the system. Most studies rather equate this composition (or the physical behavior of the cytoplasm) to that of the cryopreservation solutions applied, which is not necessarily correct. A simple extraction procedure and a general chromatographic method for small carbohydrate molecule quantification calibrated for sucrose were applied, as a first approach to work out this composition. The sucrose content data obtained are presented in Table 2, expressed as related to the total sample mass, $\operatorname{Suc}_{(\mathrm{s})}$, or to the dry mass, $\mathrm{Suc}_{(\mathrm{dm})}$, reflecting the increase in sucrose content within tissues, not only as a result of the actual increase of the content of this compound but also of the decrease in that of water.

There was a strong increase in its level between stages $b$ and $c$ (Table 2). Meanwhile, the sucrose incubation of the preculture leading to stage $b$ only implied a moderate increase (about 10\%) of the internal sucrose level; the loading with glycerol gave rise to a dramatic increase of this concentration (sevenfold). The stage $\mathrm{d}$, dehydration, after PVS2 treatment underwent a further increase, but much more moderate. This could be interpreted as a reflection of the actual cellular water content, and was in good agreement with the data of water contents and its frozen and unfrozen fraction shown in Table 1.

Other sources for changes in sucrose levels, different from the alteration in the water content, were possible, such as the actual penetration of sucrose, which could be facilitated by the presence of DMSO in the PVS2 solution, an agent known for promoting the penetration of other solutes through membranes. ${ }^{2}$

Equivalent solute concentrations (isoosmotic) can be obtained from the freezing equilibrium temperatures obtained by DSC. ${ }^{55}$ The observed decrease in this temperature can be related by Raoult's law to the molal concentration of the solution. In the case of tissues, it would rather be an average of the different solution pools in the different cell types and subcellular compartments of the specimens studied. The analytical equations are only valid for solutions much more diluted than those considered here, and their application would overestimate the solvent concentration required to induce the observed cryoscopic decrease. The $T_{\mathrm{f} \text { (peak) }}$ obtained was, so, compared with tabulated data sets, complemented when necessary with experimental DSC determinations for concentrated solutions (data not shown). Equivalent sucrose concentration, $\mathrm{Suc}_{\mathrm{eq}}$, and equivalent sodium chloride concentrations, $\mathrm{SaCl}_{\mathrm{eq}}$, were calculated and presented in $\mathrm{Ta}-$ ble 2. These coarse estimations could not describe the complex composition of cellular fluids. Nevertheless, as the observed decreases in $T_{\mathrm{f}}$ were caused by the increased number of molecules in solution, the amount of small molecular weight compounds (either dissociated as $\mathrm{NaCl}$ or not, as sucrose) would have a more determinant role than the presence of larger macromolecules and other specific plant-generated substances.

When comparing the chromatographic sucrose content with these isoosmotic solute estimated concentrations, it could be seen that the total solute content of cellular compartments must be much higher than the sucrose amount reported. Especially, a high increase in the estimated solute concentrations could be appreciated after incubation with the loading solution, may be reflecting the entrance of significant amounts of glycerol in cells, apart from the already commented dehydration. It must be noted, when comparing Table 2 parameters, that although the sucrose content chromatographically determined refers to the total or dry sample mass of the shoot tips, the estimated sucrose and sodium chloride contents, as originated from the cryoscopic decrease, refer to the solution mass (only considering solutes and solvent).

\section{General discussion}

The combined results of DSC and cryo-SEM are in good agreement, implying that part of the water contained in the system for specimens on steps $a$ and $b$ becomes frozen when cooled in LN (with less amount of ice formation in the latter), while specimens in step c would avoid ice formation, presumably getting vitrified in the cooling process, as they showed no ice trace, either microscopically or calorimetrically. This is also in good agreement with our data for viability and recovery and those of literature, resulting of applying the droplet method to several systems, including mint apices. ${ }^{11,56-64}$

The different experimental approaches used agree in a reduction of the cellular water content with the cryopreservation protocol progress. The major dehydration would take place during the loading solution incubation (stage c, showing much lower water content than b). This agrees well with the 
dry weight evolution, as well as the calorimetric frozen water content and the observed decrease of the equilibrium freezing temperature. The decrease in the size of ice crystals observed by cryo-SEM and the increases in the sucrose content obtained by ionic chromatography would confirm this result.

Regarding the last and most interesting stage $d$, there is less information available, as on one hand, the presence of large quantities of volatile substances precludes the measurement of a reliable water content by differential weighing, and on the other hand, there were no freezing events observed that could yield information about water fractions or cryoscopic decrease. Nevertheless, the sucrose content data could imply a further dehydration degree over stage c, unless a significant amount of the sucrose contained in PVS2 solution could have penetrated cells facilitated by DMSO. The evidences of vitrification derived from the cryo-SEM observations and the glass transition detected in DSC (as well as the lack of freezing or thawing events at any scanning rate) justify a higher cytoplasm viscosity in stage $d$ than in stage $c$ specimens, evidently not vitrified. This viscosity increase could be originated either by a further water content reduction or by the entrance in cells of the viscosity enhancing PVS2 components (or both).

The incubation with glycerol, a known penetrating agent, ${ }^{8,39-42}$ has been reported to promote water exit from cells, driven by the osmotic gradient, but also a substitution of part of this water by glycerol inside cells. ${ }^{39}$ The comparison of total shoot tips specimen and dry mass (Table 1) with water content data from other workers, ${ }^{8}$ whose mint shoot tip protocol is not equivalent but similar enough, allows to calculate that for stage $\mathrm{c}$ specimens, at a water content of $\sim 1.8 \mathrm{~g}_{\text {water }} \mathrm{g}_{\mathrm{dm}}{ }^{-1}$, and the amount of glycerol possible incorporated to shoot tips would be small (about $0.1 \mathrm{~g}_{\text {water }}$ $\mathrm{g}_{\mathrm{dm}}{ }^{-1}$ ). Although in this case most of the effect of the protocol step would be, then, simple osmotic dehydration, the same type of calculation applied to stage $\mathrm{d}$ yields, for a water content of $\sim 0.7 \mathrm{~g}_{\text {water }} \mathrm{g}_{\mathrm{dm}}{ }^{-1}$, an entrance of extrinsic compounds corresponding to nearly $60 \%$ of the final specimen mass (about $2.3 \mathrm{~g}_{\text {water }} \mathrm{g}_{\mathrm{dm}}{ }^{-1}$ ).

A general reflection on these data may acknowledge the natural variability of the properties determined here. In spite of the efforts to prepare equivalent start point materials and to treat them in a similar way, differences in size, permeability, microscopic scale damage, and all short of small compositional and microstructural differences would exist in a way not easy to monitor. Comparison with other similar experiments (data not shown), it can be observed that this variability would have a larger impact on the equilibrium freezing temperature data, while the enthalpy and associated water fractions would be more constant. And, an absolute observation is that for all repeats, sets of experiments, and even cooling rates applied, the final stage of the cryopreservation protocol is never producing calorimetrically detectable formation of ice. This is in good agreement with the viability results found for this protocol, ${ }^{11}$ viability that is incompatible with the formation of ice.

The calorimetric properties (melting enthalpies and equilibrium freezing and glass transition temperatures) of the cryoprotecting solutions are not too different of those of the specimens treated with them. In addition to some differences being evident, this calorimetric similarity cannot be taking as a proof of equal composition and/or behavior in other respects.
The probabilities of ice formation in specimens under $T_{\mathrm{G}}$ and over $T_{\mathrm{G}}$ but at temperatures not too elevated (roughly, no more than $20^{\circ} \mathrm{C}$ over $T_{\mathrm{G}}$ ) are similar, if a short frame time is considered, such as those corresponding to cooling or warming processes in cryopreservation. But, this may be not so for longer time periods, such as those corresponding to storage. Although storage is normally carried out below $-150^{\circ} \mathrm{C}$, well under these systems $T_{\mathrm{G}}$, it must be remembered that, when over $T_{\mathrm{G}}$, at long storage periods, ice formation is always a real possibility. ${ }^{38,65}$

A further consideration can be derived from the lack of ice observations in DSC at the last stage of the cryopreservation protocol, when the cooling is carried out at $10^{\circ} \mathrm{C} \mathrm{min}{ }^{-1}$, a rather slow speed. This, generally observed for this samples and this protocol (data not shown), would be indicative of a possible excessive amount of cryoprotecting agents, although, for the biological material used here, we find that there is no appreciable damage, at least in terms of viability and recovery. Cooling at $10^{\circ} \mathrm{C} \mathrm{min}{ }^{-1}$ would mean that the samples are in the ice formation window (roughly, without considering the differences in ice formation probabilities for different temperature regions, between -20 and $-110^{\circ} \mathrm{C}$ ) for about 9-10 min. Quench cooling would mean a permanence in this ice formation possible temperature window for only a few seconds (data not shown). Considering only the effects of the cryopreservation protocol in the formation of ice (not valuating here the possible membrane or protein protection toward low temperature or dehydration, for example) a reduction in the concentration of the vitrification solution used could produce specimens that may be iceformation prone at low cooling rates but would not form ice at quenching rates, which are those actually used in practical cryopreservation. This observation should be taken into consideration, especially when the effect of some of the cryoprotecting components of these solutions, such as DMSO, is criticized for its possible mutagenic or cytotoxic effects. ${ }^{66}$

\section{Conclusions}

Specimens of mint shoot tips at the final step of the droplet-vitrification cryopreservation protocol become vitrified and no ice is formed, which is associated to the lack of damage in the process and the resulting high viability. Meanwhile, shoot tips at previous steps of the protocol show the formation of ice by both microscopic and calorimetric observations, which is a possible cause of damage leading to the observed low viability of these specimens. The agreement between both cryo-SEM and DSC techniques is good and the potential of its associated use is clearly shown.

\section{Acknowledgments}

This work has been carried out thanks to project "CRYODYMINT" (AGL2010-21989-C02-02) of the Spanish Ministry of Science and Innovation. A.S. Teixeira was supported by the CSIC, within the JAE-Pre program, partially funded from the European Social Fund. The expert technical work of F. Pinto is also acknowledged.

\section{Literature Cited}

1. Towill LE. Cryopreservation of isolated mint shoot tips by vitrification. Plant Cell Rep. 1990;9:178-180.

2. Sakai A, Engelmann F. Vitrification, encapsulation-vitrification and droplet-vitrification: a review. CryoLetters. 2007;28:151-172. 
3. Muthusamy J, Staines HJ, Benson EE, Mansor M, Krishnapillay B. Investigating the use of fractional replication and taguchi techniques in cryopreservation: a case study using orthodox seeds of a tropical rainforest tree species. Biodivers Conserv. 2005;14:3169-3185.

4. Nadarajan J, Mansor M, Krishnapillay B, Staines HJ, Benson E, Harding K. Applications of differential scanning calorimetry in developing cryopreservation strategies for Parkia speciosa, a tropical tree producing recalcitrant seeds. CryoLetters. 2008;29:95-110.

5. Zamecnik J, Bilavcik A, Faltus M. Importance, involvement and detection of glassy state in plant meristematic tissue for cryopreservation. Cryobiology. 2007;5:324-378.

6. Niino T, Sakai A, Yakuwa H, Nojiri K. Cryopreservation of in vitro-grown shoot tips of apple and pear by vitrification. Plant Cell Tissue Organ Cult. 1992;28:261-266.

7. Reinhoud PJ. Cryopreservation of tobacco suspension cells by vitrification. Doctoral Paper, Rijks University, Leiden, The Netherlands, 1996.

8. Volk GM, Walters C. Plant vitrification solution 2 lowers water content and alters freezing behavior in shoot tips during cryoprotection. Cryobiology. 2006;52:48-61.

9. Wesley-Smith J, Walters C, Pammenter NW, Berjak P. Interactions among water content, rapid (nonequilibrium) cooling to $-196^{\circ} \mathrm{C}$, and survival of embryonic axes of Aesculus hippocastanum L. seeds. CryoLetters. 2001;42:196-206.

10. Kauzmann W. The nature of the glassy state and the behavior of liquids at low temperatures. Chem Rev. 1948;43:219-256.

11. Senula A, Joachim Keller ER, Sanduijav T, Yohannen T. Cryopreservation of cold-acclimated mint (Mentha spp.) shoot tips using a simple vitrification protocol. CryoLetters. 2007;28:1-12.

12. Kartha $\mathrm{KK}$, Leung $\mathrm{NL}$, Mroginski LA. In vitro growthresponses and plant-regeneration from cryopreserved meristems of cassava (Manihot esculenta Crantz), $Z$ Pflanzenphysiol. 1982;107:133-140.

13. Sakai A, Kobayashi S, Oiyama I. Cryopreservation of nucellar cell of navel orange (Citrus sinensis Obs. var. brasiliensis Tanaka) by vitrification. Plant Cell Rep. 1990;9:30-33.

14. Reed BM. Plant Cryopreservation: A Practical Guide. New York: Springer; 2008.

15. Dereuddre J, Scottez C, Arnaud Y, Duron M. Résistance d'apex caulinaires de vitroplants de Poirier (Pyrus communis L. cv Beurré Hardy), enrobés dans l'alginate, à une déshydratation puis à une congélation dans l'azote liquide: effet d'un endurcissement préalable au froid. C R Acad Sci. 1990;10:317-323.

16. Jouve L, Hoffmann L, Hausman JF. Polyamine, carbohydrate, and proline content changes during salt stress exposure of aspen (Populus tremula L.): involvement of oxidation and osmoregulation metabolism. Plant Biol. 2004;6:74-80.

17. Fahy GM, MacFarlane DR, Angell CA, Meryman HT. Vitrification as an approach to cryopreservation. Cryobiology. 1984;21:407-426.

18. Benson EE. An introduction to plant conservation biotechnology. In: Benson EE, editor. Plant Conservation Biotechnology. London: Taylor \& Francis; 1999:3-10.

19. Suzuki M, Ishikawa M, Okuda H, Noda K, Kishimoto T, Nakamura T, Ogiwara I, Shimura I, Akihama T. Physiological changes in gentian axillary buds during two-step preculturing with sucrose that conferred high levels of tolerance to desiccation and cryopreservation. Ann Bot. 2006;97:1073-1081.

20. Carpenter JF, Dawson PE. Quantification of dimethyl sulfoxide in solutions and tissues by high performance liquid chromatography. Cryobiology. 1991;28:210-215.

21. Cheng X, Kaplan LA. Simultaneous analyses of neutral carbohydrates and amino sugars in freshwaters with HPLC-PAD. $J$ Chromatogr Sci. 2003;41:434-438.

22. Lee YC. Carbohydrate analyses with high-performance anionexchange chromatography. $J$ Chromatogr A. 1996;720:137-149.

23. Masuda T, Kitahara K, Aikawa Y, Arai S. Determination of carbohydrates by HPLC-ECD with a novel stationary phase prepared from polystyrene-based resin and tertiary amines. $J \mathrm{Am}$ Chem Soc. 2001;17:895-898.
24. Schiller M, von der Heydt H, März F, Schmidt P. Quantification of sugars and organic acids in hygroscopic pharmaceutical herbal dry extracts. $J$ Chromatogr A. 2002;968:101-111.

25. Matsumoto T, Sakai A, Yamada K. Cryopreservation of in-vitro grown apical meristems of wasabi (Wasabia japonica) by vitrification and subsequent high plant-regeneration. Plant Cell Rep. 1994; 13:442-446.

26. Yamada T, Sakai A, Matsumura T, Higuchi S. Cryopreservation of apical meristems of white clover (Trifolium repens L.) by vitrification. Plant Sci. 1991;78:81-87.

27. González-Benito ME, Mendoza-Condori VH, Molina-García AD. Cryopreservation of in vitro shoot apices of Oxalis tuberosa Mol. CryoLetters. 2007;28:23-32.

28. Craig $S$, Beaton CD. A simple cryo-SEM method for delicate plant tissues. J Microsc. 1996;182:102-105.

29. Murashige T, Skoog F. A revised medium for rapid bioassays with tobacco tissue cultures. Physiol Plantarum. 1962;15:473497.

30. Fullerton GD, Keener CR, Cameron IL. Correction for solute/ solvent interaction extends accurate freezing point depression theory to high concentration range. J Biochem Biophys Methods. 1994;29:217-235.

31. Elliott JAW, Prickett RC, Elmoazzen HY, Porter KR, McGann LE. A multisolute osmotic virial equation for solutions of interest in biology. $J$ Phys Chem B. 2007;111:1775-1785.

32. Guignon B, Torrecilla JS, Otero L, Ramos AM, Molina-García $\mathrm{AD}$, Sanz PD. The initial freezing temperature of foods at high pressure. Crit Rev Food Sci Nutr. 2008;48:328-340.

33. Wolf AV, Brown MG, Prentiss PG. Concentrative properties of aqueous solutions. In: Weast RC, editor. Handbook of Chemistry and Physics. Boca Raton: CRC Press; 1985:D-219-D-271.

34. Otero L, Molina-García AD, Sanz PD. Some interrelated thermophysical properties of liquid water and ice. I. A user-friendly modelling review for food high-pressure processing. Crit Rev Food Sci Nutr. 2002;42:339-352.

35. Angell CA, Shuppert J, Tucker JC. Anomalous properties of supercooled water. Heat capacity, expansivity, and proton magnetic resonance chemical shift from 0 to $-38^{\circ}$.J Phys Chem. 1973;77:3892-3899.

36. Angell CA, Ogunl M, Slchlna WJ. Heat capacity of water at extremes of supercooling and superheating. J Phys Chem. 1982;86:998-1002.

37. Umrath W. Calculation of the freeze-drying time for electronmicroscopical preparations. Mikroskopie. 1983;40:9-34.

38. Franks F. Metastable water at subzero temperatures. J Microsc. $1986 ; 141: 243-249$

39. Benson EE. Cryopreservation theory. In: Reed BM, editor. Plant Cryopreservation: A Practical Guide. New York: Springer; 2008:15-32.

40. Schäfer-Menhur A, Schumacher HM, Mix-Wagner G. Cryopreservation of potato cultivars-design of a method for routine application in genebanks. Acta Hort. 1997;447:477-482.

41. Hubálek Z. Protectants used in the cryopreservation of microorganisms. Cryobiology. 2003;46:205-229.

42. Panis B, Lombardi M. Status of cryopreservation technologies in plants (food and forest crops). In: Ruane J, Sonnino A, editors. The Role of Biotechnology in Exploring and Protecting Agricultural Genetic Resources. Rome: FAO; 2006:61-78.

43. Robinson W. Free and bound water determinations by the heat of fusion of ice method. $J$ Biol Chem. 1931;92:699-709.

44. Franks F. Unfrozen water - yes - unfreezable water - hardly bound water - certainly not. CryoLetters. 1986;7:207.

45. Hills BP, Takacs SF, Belton PS. A new interpretation of proton NMR relaxation time measurements of water in food. Food Chem. 1990;37:95-111.

46. Wiggins PM. Role of water in some biological processes. Microbiol Rev. 1990;54:432-449.

47. Hills BP, Cano C, Belton PS. Proton NMR relaxation studies of aqueous polysaccharide systems. Macromolecules. 1991;1:29442950.

48. Slade L, Levine H, Reid DS. Beyond water activity: recent advances based on an alternative approach to the assessment of food quality and safety. Crit Rev Food Sci Nutr. 1991;30:115360 
49. Hills BP, Tang H, Belton PS, Khaliq A, Harris RK. NMR Oxygen-17 studies of the state of water in a saturated sucrose solution. J Mol Liq. 1998;75:45-59.

50. Wolfe $\mathbf{J}$, Bryant G, Koster KL. What is 'unfreezable water', how unfreezable is it and how much is there? CryoLetters. 2002;23:157-166.

51. Obbard R, Iliescu D, Cullen D, Chang J, Baker I. SEM/EDS comparison of polar and seasonal temperate ice. Microsc Res Tech. 2003;62:49-61.

52. Ohnoa H, Igarashib M, Hondota T. Salt inclusions in polar ice core: location and chemical form of water-soluble impurities. Earth Planet Sci Lett. 2005;232:171-178.

53. Hashimoto T, Tasaki Y, Harada M, Okada T. Electrolyte-doped ice as a platform for atto- to femtoliter reactor enabling zeptomol detection. Anal Chem. 2011;83:3950-3956.

54. Verdonck E, Schaap K, Thomas L. A discussion of the principles and applications of modulated temperature DSC (MTDSC). Int J Pharm. 1999;192:3-20.

55. Buckley KA, Conway EJ, Ryan HC. Concerning the determination of total intracellular concentrations by the cryoscopic method. I Physiol. 1958;143:236-245.

56. Keller ERJ, Dreiling M. Potato cryopreservation in Germanyusing the droplet method for the establishment of a new large collection. Acta Hort. 2003;623:193-200.

57. Halmagyi A, Deliu C, Coste A. Plant regrowth from potato shoot tips cryopreserved by a combined vitrification-droplet method. CryoLetters. 2005;26:313-322.

58. Leunufna S, Keller ERJ. Cryopreservation of yams using vitrification modified by including droplet method: effects of cold acclimation and sucrose. CryoLetters. 2005;26:93-102.
59. Panis B, Piette B, Swennen R. Droplet vitrification of apical meristems: a cryopreservation protocol applicable to all $\mathrm{Musa}$ ceae. Plant Sci. 2005;168:45-55.

60. Kim HH, Lee JK, Yoon JW, Ji JJ, Nam SS, Hwang HS, Cho EG, Engelmann F. Cryopreservation of garlic bulbil primordia by the droplet-vitrification procedure. CryoLetters. 2006;27:143153.

61. Kryszczuk A, Keller J, Grübe M, Zimnoch-Guzowska E. Cryopreservation of potato (Solanum tuberosum L.) shoot tips using vitrification and droplet method. Food Agric Environ. 2006;4:196-200.

62. Sant R, Panis B, Taylor M, Tyagi A. Cryopreservation of shoottips by droplet vitrification applicable to all taro (Colocasia esculenta var. esculenta) accessions. Plant Cell Tissue Organ Cult. 2008;92:107-111.

63. Halmagyi A, Deliu C, Isac V. Cryopreservation of Malus cultivars: comparison of two droplet protocols. Sci Hortic. 2010;124:387-392.

64. Vujovic T, Sylvestre I, Ruzic D, Engelmann F. Droplet-vitrification of apical shoot tips of Rubus fruticosus L. and Prunus cerasifera Ehrh. Sci Hortic. 2011;130:222-228.

65. Angell CA. Supercooled water. In: Franks F, editor. Water-A Comprehensive Treatise, Vol. 7. New York: Plenum Press; 1982:215-338.

66. Harding K. Genetic integrity of cryopreserved plant cells: a review. CryoLetters. 2004;18:217-230.

Manuscript received Oct. 26, 2012, and revision received Jan. 11, 2013. 